Published in final edited form as:

ACS Nano. 2019 September 24; 13(9): 10490-10498. doi:10.1021/acsnano.9b04453.

\title{
Controlled Growth of Large-Area Bilayer Tungsten Diselenides with Lateral $P-N$ Junctions
}

\author{
Srinivas V. Mandyam ${ }^{\perp, \dagger}$, Meng-Qiang Zhao ${ }^{\perp, \dagger}$, Paul Masih Das $^{\dagger}$, Qicheng Zhang $^{\dagger}$, \\ Christopher C. Price ${ }^{\S}$ Zhaoli Gao ${ }^{\dagger}$, Vivek B. Shenoy ${ }^{\S}$ Marija Drndić ${ }^{\dagger}$, Alan T. Charlie \\ Johnson ${ }^{\dagger,}$ \\ †Department of Physics and Astronomy, University of Pennsylvania, 209 South $33^{\text {rd }}$ Street, \\ Philadelphia, PA 19104, USA \\ §Department of Materials Science and Engineering, University of Pennsylvania, 3231 Walnut St., \\ Philadelphia, PA 19104, USA
}

\begin{abstract}
Bilayer two-dimensional (2D) van der Waals ( $\mathrm{vdW}$ ) materials are attracting increasing attention due to their predicted high quality electronic and optical properties. Here we demonstrate dense, selective growth of $\mathrm{WSe}_{2}$ bilayer flakes by chemical vapor deposition with the use of a 1:10 molar mixture of sodium cholate and sodium chloride as the growth promoter to control the local diffusion of $\mathrm{W}$-containing species. A large fraction of the bilayer $\mathrm{WSe}_{2}$ flakes showed a $0(\mathrm{AB})$ and $60^{\circ}$ (AA') twist between the two layers, while moiré 15 and $30^{\circ}$-twist angles were also observed. Well-defined monolayer-bilayer junctions were formed in the as-grown bilayer $\mathrm{WSe}_{2}$ flakes, and these interfaces exhibited $p$ - $n$ diode rectification and an ambipolar transport characteristic. This work provides an efficient method for the layer-controlled growth of $2 \mathrm{D}$ materials, in particular, 2D transition metal dichalcogenides and promotes their applications in next-generation electronic and optoelectronic devices.
\end{abstract}

\section{Keywords}

two-dimensional materials; controlled growth; bilayer $\mathrm{WSe}_{2}$; growth promoter; monolayer-bilayer junction

Two-dimensional (2D) van der Waals (vdW) materials fabricated by stacking of homogeneous or heterogeneous monolayer "building blocks" are attracting increasing

\footnotetext{
*Corresponding Author: cjohnson@ physics.upenn.edu.

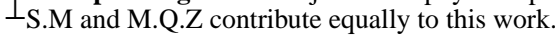

Author Contributions

The manuscript was written through contributions of all authors. All authors have given approval to the final version of the manuscript.

The authors declare no competing financial interest.

Supporting Information

The Supporting Information is available free of charge on the ACS Publications website at DOI: 10.1021/acsnano.9b04453.

Optical images of the as-grown WSe 2 flakes using different growth promoters and partially oxidized bilayer $\mathrm{WSe}_{2}$; Characterizations of the as-grown bilayer WS2 flakes; Analysis of the stacking orientation and atomic structure of bilayer WSe2; SAED patterns of the bilayer WSe2 and corresponding statistical analysis; Atomic models of bilayer $\mathrm{WSe}_{2}$ with varied stacking orientations;

Thermodynamic growth diagram for bilayer WSe 2 flakes; Characterization data of a $\mathrm{WSe}_{2}$ FET based on the monolayer region.
} 
attention, since their physical and chemical properties offer promise for use in atomically thin electronic and optoelectronic devices. ${ }^{1-9}$ For example, vertical stacking of $2 \mathrm{D} \mathrm{MoS}_{2}$ and $\mathrm{WSe}_{2}$ flakes leads to the formation of atomically thin $p$ - $n$ heterojunctions. ${ }^{10,11} \mathrm{~A}$ stack of two graphene layers at a 'magic' twist angle exhibits flat electronic energy bands near the Fermi energy, resulting in correlated insulating states at half-filling and unconventional superconductivity upon electrostatic doping of the material away from the correlated insulating state. ${ }^{12,13}$

For stacks of homogeneous 2D vdW materials, the number of layers plays a crucial role in the ultimate optoelectronic properties. ${ }^{1,14-17}$ Since the change in properties from monolayer to bilayer is typically more significant than that resulting from additional layers, precise control over bilayer formation is of great interest. ${ }^{18-21}$ For example, for many $2 \mathrm{D}$ transition metal dichalcogenides (TMDs), the bandgap is direct for the monolayer and indirect for the bilayer. ${ }^{16,22,23}$ Also bilayer graphene has a widely tunable bandgap unlike the monolayer. ${ }^{24}$ Note that the band offsets between different TMD materials are significant, which could inhibit carrier transport. ${ }^{25}$ In contrast, homogeneous bilayer TMDs are expected to exhibit superior performance for applications in optoelectronic devices such as light-emitting diodes, laser diodes, and solar cells. ${ }^{18,26-28}$

A variety of approaches have been developed to create bilayer TMDs, including mechanical exfoliation from the bulk, ${ }^{29}$ mechanical transfer, ${ }^{21,} 30$ drop casting ${ }^{31}$ or layer-by-layer assembly ${ }^{32}$ using exfoliated suspensions, and chemical vapor deposition (CVD). ${ }^{1}, 14,18$ CVD is of particular interest because it not only provides high-quality and scalable TMDs but also can lead to cleaner interfaces between the layers and more intimate electronic coupling. The TMD layer number is affected by many CVD growth parameters, such as substrate surface chemistry, ${ }^{33-34}$ partial pressure of the precursors, ${ }^{35,} 36$ and growth temperature. ${ }^{18,37}$ For example, layer-controlled CVD growth of large-area $\mathrm{MoS}_{2}$ films (including bilayer) was achieved by precise control of the $\mathrm{MoCl}_{5}$ precursor ${ }^{35}$ and by treating the $\mathrm{SiO}_{2}$ substrate surface with an oxygen plasma. ${ }^{33} \mathrm{CVD}$ growth of TMD flakes with $1-4$ layers has been achieved through the choice of growth temperature. ${ }^{18,37}$ In contrast to largearea continuous films, TMD flakes can contain both monolayer (1L) and bilayer (2L) regions, and the $1 \mathrm{~L}-2 \mathrm{~L}$ junction exhibits appealing optoelectronic properties for further device applications. ${ }^{26-28}$ So far, reported methods for synthesis of bilayer TMD flakes have resulted in a mixture of monolayer, bilayer, and few-layer flakes, with no more than $80 \%$ bilayer content. ${ }^{18,37,38}$ Obtaining efficient growth of high-density homogeneous bilayer TMD flakes with well-defined 1L-2L junctions remains a challenge.

The complex growth environment during CVD complicates precise control over the final TMD structure. Recently, a multiscale model for vertical growth of $2 \mathrm{D} v \mathrm{vdW}$ materials was proposed that indicated that temperature and vapor adatom flux are the primary parameters affecting the final TMD structure, in agreement with experiments. ${ }^{20}$ Among the TMDs, 2D $\mathrm{WSe}_{2}$ is of particular interest because of its tunable transport behaviors and suitability for a variety of electronic and optoelectronic applications. ${ }^{37,39} \mathrm{Here}$, we report an approach for large-area growth of high-density, almost purely bilayer $\mathrm{WSe}_{2}$ flakes using a mixture of sodium cholate and sodium chloride as the growth promoter. These bilayer $\mathrm{WSe}_{2}$ flakes provide abundant lateral $1 \mathrm{~L}-2 \mathrm{~L}$ junctions which exhibit promising $p$ - $n$ diode-like 
rectification behavior, suggesting their potential use in atomically thin electronics and optoelectronics.

\section{RESULTS AND DISCUSSION}

A variety of organic molecules and salts have been explored as growth promoters of TMDs. 40, 41 Organic aromatic molecules are expected to enhance the wettability of the growth substrate and lower the free energy for nucleation, which should promote adsorption of transition metal (TM) oxide precursors on the substrate and thus growth of monolayer flakes. ${ }^{40,42}$ In contrast, alkali metal halides can react with TM oxides to form volatile oxyhalides, which promotes the escape of TM precursors into the vapor and facilitates the vertical growth of additional TMD layers. ${ }^{20,43,44} \mathrm{We}$ discovered that a suitable balance of these two competing mechanisms enabled tuning of the layer number in CVD-grown TMD flakes,${ }^{20}$ as illustrated in Figure 1. In our growth method, W precursor is pre-deposited on the substrate by spin-coating of an ammonium metatungstate aqueous solution, together with the growth promoters (see details in Methods). When using sodium cholate $\left(\mathrm{C}_{24} \mathrm{H}_{40} \mathrm{NaO}_{5}\right)$ as the growth promoter, the aromatic cholate anions enhance the adsorption of $\mathrm{WO}_{\mathrm{x}}$ tungsten precursors onto the substrate and deplete $\mathrm{W}$ adatom flux in the vapor, leading to preferential growth of monolayer $\mathrm{WSe}_{2}$ (Figure 1a). ${ }^{20,40,42}$ The addition of $\mathrm{NaCl}$ transforms some $\mathrm{WO}_{\mathrm{x}}$ into oxyhalide $\left(\mathrm{WO}_{\mathrm{x}} \mathrm{Cl}\right)$, which is more easily evaporated at high temperature, leading to increased $\mathrm{W}$ adatom flux from the vapor and enhanced growth of the bilayer (Figure 1b) ${ }^{20,43,44}$ When pure $\mathrm{NaCl}$ is used as the growth promoter, a large amount of $\mathrm{WO}_{\mathrm{x}} \mathrm{Cl}$ forms, leading to reduced surface adsorption and further increased $\mathrm{W}$ vapor adatom flux, so that multilayer (ML) $\mathrm{WSe}_{2}$ dominates the final structures (Figure 1c).

Figure 2 shows optical micrographs of $\mathrm{WSe}_{2}$ flakes grown using these three different promoter mixtures. The bare substrate and TMD flake regions with different layer numbers in these images are readily identified due to differences in optical contrast. ${ }^{45}$ When $25 \mathrm{mM}$ $\mathrm{C}_{24} \mathrm{H}_{40} \mathrm{NaO}_{5}$ was used as the growth promoter, star-like $\mathrm{WSe}_{2}$ flakes were obtained that were nearly exclusively monolayer (Figure $2 \mathrm{a}-\mathrm{b}$ ). Vertical growth of $\mathrm{WSe}_{2}$ was induced by adding $\mathrm{NaCl}$ to allow for enhanced $\mathrm{W}$ diffusion from the vapor. When the promoter solution contained $25 \mathrm{mM}$ sodium cholate and $125 \mathrm{mM} \mathrm{NaCl}$, the growth product evolved into monolayer $\mathrm{WSe}_{2}$ with small bilayer regions (Figure S1a). When the promoter solution contained $25 \mathrm{mM} \mathrm{C}_{24} \mathrm{H}_{40} \mathrm{NaO}_{5}$ and $250 \mathrm{mM} \mathrm{NaCl}$, the growth was optimized and yielded predominantly bilayer $\mathrm{WSe}_{2}$ with sharp $1 \mathrm{~L}-2 \mathrm{~L}$ boundaries (Figure $2 \mathrm{c}-\mathrm{d}$ ).

After examining $>500$ flakes over an area of $1 \times 2 \mathrm{~cm}^{2}$, it was confirmed that $100 \%$ of the flakes were bilayer $\mathrm{WSe}_{2}$ with a high surface coverage of $67.8 \pm 1.5 \%$ and a size ranging from $40-80 \mu \mathrm{m}$. No monolayer $\mathrm{WSe}_{2}$ flakes were observed, and only a few multilayer regions (bright spots in Figure 2c) were found, which was attributed to non-uniform distribution of $\mathrm{W}$ precursor. The formation of six-point star-like morphology of bilayer $\mathrm{WSe}_{2}$ was ascribed to a higher Se:W ratio during the growth compared to the level that leads to conventional triangular or hexagonal morphologies. ${ }^{46,47}$ These star-like flakes are composed of six diamond-shaped single crystals with mirror grain boundaries (GBs) between each other, as confirmed by optical microscopy after partial oxidation to reveal the

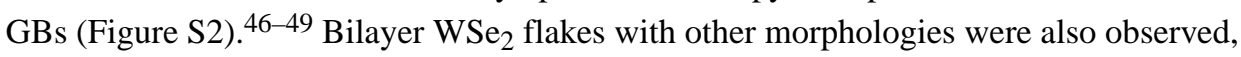


indicating that increasing the local value of the Se:W ratio induced a transformation from triangular to star-like flakes (Figure S3). ${ }^{47}$ When the $\mathrm{NaCl}$ concentration was further increased to $500 \mathrm{mM}$, a large amount of $\mathrm{ML} \mathrm{WSe}_{2}$ regions were observed (Figure $\mathrm{S} 1 \mathrm{~b}$ ).

To demonstrate the importance of the $\mathrm{C}_{24} \mathrm{H}_{40} \mathrm{NaO}_{5}$ component, we conducted the growth with a $250 \mathrm{mM}$ solution of $\mathrm{NaCl}$ as the promoter and observed the formation of multiple bilayer and multilayer regions on large monolayer flakes (Figure 2e-f). This result can be explained by greatly enhanced diffusion of $\mathrm{W}$ from the vapor due to the formation of oxyhalides and comparatively inefficient surface adsorption due to the absence of cholate ions. We also observed that the flakes were largely hexagonal rather than star-like, indicating a lower Se:W ratio. ${ }^{47}$ The $\mathrm{ML} \mathrm{WSe}_{2}$ regions became smaller with decreasing $\mathrm{NaCl}$ concentration. When the $\mathrm{NaCl}$ concentration was reduced to $75 \mathrm{mM}$, the growth yielded a large amount of randomly distributed $2 \mathrm{~L}$ regions on large $1 \mathrm{~L} \mathrm{WSe}{ }_{2}$ flakes (Figures $\mathrm{S} 4 \mathrm{a}-\mathrm{b}$ ). At $25 \mathrm{mM}$ of $\mathrm{NaCl}$, the growth consisted nearly exclusively of monolayer star-shape $\mathrm{WSe}_{2}$ flakes decorated with a high density of small $2 \mathrm{~L}$ regions (Figures $\mathrm{S} 4 \mathrm{c}-\mathrm{d}$ ). The growth experiments done with varied $\mathrm{NaCl}$ concentrations indicated that the use of pure $\mathrm{NaCl}$ as a promoter cannot lead to the growth of high-quality bilayer $\mathrm{WSe}_{2}$ flakes with large, continuous $2 \mathrm{~L}$ regions. This revealed the essential role of surface adsorption introduced by cholate ions in the controlled growth of bilayer $\mathrm{WSe}_{2}$. Note that all the above mentioned morphologies for the as-grown $\mathrm{WSe}_{2}$ also showed a selectivity of $\sim 100 \%$ based on the statistical analysis of $>500$ flakes over an area of $1 \times 2 \mathrm{~cm}^{2}$. We further applied the bilayer growth method to other TMDs. As shown in Figures S5, the use of $25 \mathrm{mM} \mathrm{C}_{24} \mathrm{H}_{40} \mathrm{NaO}_{5}$ and $250 \mathrm{mM} \mathrm{NaCl}$ led to the preferential growth of bilayer $\mathrm{WS}_{2}$ while only monolayer flakes were obtained with $25 \mathrm{mM} \mathrm{C}_{24} \mathrm{H}_{40} \mathrm{NaO}_{5}$ as the growth promoter.

Atomic force microscopy (AFM) was used to confirm the assignment of monolayer and bilayer $\mathrm{WSe}_{2}$ flakes. A low-magnification AFM image is shown in Figure 3a, which reveals distinct $1 \mathrm{~L}$ and $2 \mathrm{~L}$ regions. The step heights at the substrate-to- $1 \mathrm{~L}$ and $1 \mathrm{~L}-$ to- $2 \mathrm{~L}$ interfaces were measured to be $0.83 \pm 0.09$ and $0.81 \pm 0.08 \mathrm{~nm}$, respectively, as expected for bilayer material (Figure 3b-c). Raman and photoluminescence (PL) spectroscopy were also used to confirm the assignment. Raman spectra collected from the $1 \mathrm{~L}$ region of a bilayer $\mathrm{WSe}_{2}$ flake showed peaks at 248 and $260 \mathrm{~cm}^{-1}$, which correspond to the $\mathrm{E}_{2 \mathrm{~g}}^{1} / \mathrm{A}_{1 \mathrm{~g}}$ and $2 \mathrm{LA}(\mathrm{M})$ modes of the monolayer, respectively. For the inner $2 \mathrm{~L}$ region, these two peaks red-shifted to 250 and $263 \mathrm{~cm}^{-1}$ (Figure $3 \mathrm{~d}$ ), in good agreement with previous reports. ${ }^{50}$ The distribution of $1 \mathrm{~L}$ and $2 \mathrm{~L}$ regions was further examined by Raman intensity maps at 248 and $250 \mathrm{~cm}^{-1}$. A clear 1L-2L boundary was observed in the combined Raman intensity map shown in Figure 3e, which agreed well with the optical image shown in Figure 2d. The PL spectrum (Figure 3f) acquired from the $1 \mathrm{~L}$ region of a bilayer $\mathrm{WSe}_{2}$ flake showed a strong peak at an energy of $1.66 \mathrm{eV}$ (wavelength of $749 \mathrm{~nm}$ ), characteristic of a direct excitonic transition. In contrast, on the $2 \mathrm{~L}$ region the prominent peak showed a lower intensity, indicative of an indirect bandgap transition, and it was shifted to an excitonic transition energy of $1.57 \mathrm{eV}$ (wavelength of $791 \mathrm{~nm}$ ). ${ }^{51}$

To investigate the atomic scale structure of bilayer $\mathrm{WSe}_{2}$, scanning transmission electron microscopy (STEM) was employed. STEM high-angle annular dark field (STEM-HAADF) images are widely used to identify detailed atomic arrangement of crystalline structures 
because its contrast is sensitive to the atomic number and sample thickness. ${ }^{52}$ Figure $4 \mathrm{a}$ shows a low-magnification STEM image of a $1 \mathrm{~L}-2 \mathrm{~L}$ boundary region of an as-grown $\mathrm{WSe}_{2}$ flake, with an atomically sharp $1 \mathrm{~L}-2 \mathrm{~L}$ interface (Figure $4 \mathrm{~b}$ ). The usual hexagonal arrangement of Se and $\mathrm{W}$ atoms, corresponding to the $1 \mathrm{H}$ phase, is clearly shown in the $1 \mathrm{~L}$ region. The $2 \mathrm{~L}$ region has a $60^{\circ}$-twist angle, as confirmed by the SAED pattern (Figure $4 \mathrm{c}$ ) and fast Fourier transform (FFT) decomposition (Figures S6a-c), with the resulting atomic model shown in Figure 4d. Disordered hexagonal shapes at the 1L-2L boundary are observed, which is ascribed to strain introduced by interlayer vdW interactions.

The atomic structure of a $1 \mathrm{~L}-2 \mathrm{~L}$ boundary region with a $15^{\circ}$-twist angle is shown in Figure $4 \mathrm{e}$, and the twist angle is confirmed by SAED (Figure $4 \mathrm{f}$ ) of the $2 \mathrm{~L}$ region. The $15^{\circ}$ twist angle leads to the formation of moire patterns, as seen in the STEM-HAADF image and the atomic model of Figure 4g. Examples of $\mathrm{AB}$ stacking $\left(0^{\circ}\right.$ twist) were also observed (Figures $\mathrm{S} 6 \mathrm{~g}-\mathrm{i})$. As mentioned above, star-like $\mathrm{WSe}_{2}$ flakes are composed of six diamond-like single crystals separated by GBs. The atomic structure of one such GB is shown in Figure 4h, where neighboring $2 \mathrm{~L}$ regions exhibit twist angles of 15 and $60^{\circ}$. Although part of the GB was damaged during transfer, which exposed the bottom $1 \mathrm{~L}$ region, seamless connection between the top neighboring $\mathrm{WSe}_{2}$ layers is still observed. This STEM image clearly reveals that different twist angles exist in a single as-grown bilayer $\mathrm{WSe}_{2}$ flake. The stacking orientation of two TMD layers can be used to manipulate their electrical, optical, and vibrational properties. ${ }^{21,53}$

We conducted a SAED survey of $\sim 100$ bilayer flakes and found that only twist angles of $0^{\circ} / 60^{\circ}$ (these twist angles cannot be differentiated with SAED alone), $15^{\circ}$, and $30^{\circ}$ were represented (Figure S7). A twist angle of $0^{\circ} / 60^{\circ}$ could indicate any of three distinct stacking structures: AA $\left(0^{\circ}\right.$; known to be thermodynamically unstable $\left.{ }^{54-56}\right), \mathrm{AB}\left(0^{\circ}\right)$, and $\mathrm{AA}^{\prime}\left(60^{\circ}\right)$. The strong majority $(\sim 84 \%)$ of flakes showed a twist angle of $0^{\circ} / 60^{\circ}$, with smaller fractions having a twist of $30^{\circ}(12 \%)$ or $15^{\circ}(4 \%)$. A summary of the twist angles and corresponding atomic models is provided in Figure S8. Due to the complex growth environment, a good control over the twist angles between TMD layers by CVD is still a challenge.

We examined the energetics of different bilayer orientations using our previously developed thermodynamic criterion for bilayer CVD growth of TMDs: ${ }^{20} \varepsilon_{\mathrm{L} 1: \mathrm{S}}-\varepsilon_{\mathrm{L} 1: \mathrm{L} 2}>\eta(\gamma / \mathrm{L} 2-\gamma /$ L1). Here, $\varepsilon_{\mathrm{L} 1: \mathrm{L} 2}$ is the TMD stacking energy, $\varepsilon_{\mathrm{L} 1: \mathrm{S}}$ is the TMD/substrate interaction energy, $\gamma$ is the $\mathrm{WSe}_{2}$ Se-terminated zig-zag edge energy, $\eta=4 / 3^{1 / 2}$ is a geometric factor for diamond flakes, and L1, L2 are the edge lengths of the bottom and top layers, respectively. This criterion determines whether additional growth of the top layer is thermodynamically favorable based on the sizes of the bottom and top layers (constrained such that L2 < L1), and the energetics of edge formation and stacking of the TMD layers. We chose diamond flakes to match the experimental observations, and we calculated the Na-doped Seterminated zigzag edge energy to be $0.57 \mathrm{eV} / \AA$. Figure $\mathrm{S} 9$ shows a plot of the criteria over the values of $\mathrm{L} 1$ and $\mathrm{L} 2$ for the different rotational alignments of the $\mathrm{WSe}_{2}$ bilayer observed during growth. We see that the minimum size for a stable bilayer nucleus to grow is $\sim 1.5$ $\mathrm{nm}$, corresponding to a 2D triangular flake containing at least $40 \mathrm{~W}$ atoms. Furthermore, critical thermodynamic stability of the top layer (independent of the bottom layer size) is not reached until the top layer flake reaches $4 \mathrm{~nm}$ for the $\mathrm{AA}^{\prime}$ and $\mathrm{AB}$ stacking orientations, and 
$6 \mathrm{~nm}$ for the $15^{\circ}$ and $30^{\circ}$ orientation. In between the minimum size and the critical size, the morphology will be kinetically determined, which agrees with the experimental finding that altering the metal diffusion rate through promoter composition determines the preference for bilayer vs. monolayer. In particular, increasing the metal diffusivity (through introduction of volatile halide anions) will increase the kinetic probability of bilayer morphology. We also found that the $60^{\circ}\left(\mathrm{AA}^{\prime}\right)$ and $0^{\circ}(\mathrm{AB})$ stacking energies are the most favorable and almost identical (Table 1), leading to the prediction that they should dominate the population. The next most favorable stackings are the 30 and $15^{\circ}$ twisted structures, consistent with their lower observed frequency in the growth. High energy stacking structures, such as $0^{\circ}$ (AA), have very large thermodynamic critical sizes $(10 \mathrm{~nm})$, consistent with the fact that they are not observed in the experiments.

A set of back-gated field effect transistors (FETs), with electrodes 1-5, was fabricated across the $1 \mathrm{~L}-2 \mathrm{~L}$ domains as well as GBs within a single bilayer flake, as shown in Figures 5a-b. The $\mathrm{I}_{\mathrm{ds}}-\mathrm{V}_{\mathrm{ds}}$ curves of the $1 \mathrm{~L}-2 \mathrm{~L}$ junction (electrodes $\left.1 / 2\right)$ at different gate voltages $\left(\mathrm{V}_{\mathrm{g}}\right)$ exhibited typical rectification of a $p-n$ diode structure (Figure $5 \mathrm{c}$ ), which is ascribed to the different built-in surface potentials of $1 \mathrm{~L}$ and $2 \mathrm{~L} \mathrm{WSe} e_{2}$ and band structure offsets at their interface. $^{2,27}$ The formation of $p$ - $n$ diodes with 1L-2L TMD junctions has been widely reported. ${ }^{2,18,27}$ In particular, the band structure of $\mathrm{WSe}_{2} 1 \mathrm{~L}-2 \mathrm{~L}$ junctions and the mechanism for the rectification behavior have been documented, and our results are in good agreement with this report. ${ }^{27}$ With a gate voltage of $-80 \mathrm{~V}$ and a forward (reverse) bias of 4 $\mathrm{V}$, the device current is $5.4 \mu \mathrm{A}(-0.4 \mu \mathrm{A})$, yielding a rectification ratio of 13.5 . Figure $5 \mathrm{~d}$ presents the $\mathrm{I}_{\mathrm{ds}}-\mathrm{V}_{\mathrm{g}}$ transfer characteristic of the same device for different source-drain voltages. The transfer curves exhibit ambipolar behavior, but the conductance in the $p$ branch is about 1000 times larger than in the $n$-branch. This is contributed by both the higher hole mobilities compared to electron for $\mathrm{WSe}_{2}$ and the existence of current transport barrier on the $n$-branch of the $p$ - $n$ diode device. ${ }^{37,39,57}$

We further investigated the electrical transport behavior of monolayer and bilayer $\mathrm{WSe}_{2}$ by measuring devices defined by the electrode pairs 2/3 (S2D3; a bilayer device), 2/4 (S2D4; bilayer device including a GB), and 1/5 (S1D5; monolayer device including a GB). As shown in Figure 5e, the $\mathrm{I}_{\mathrm{ds}}-\mathrm{V}_{\mathrm{g}}$ curves for all three FET devices exhibited $p$-type behavior. The carrier mobility of FET S2D3 was calculated as $7.9 \mathrm{~cm}^{2} \mathrm{~s}^{-1} \mathrm{~V}^{-1}$, somewhat higher than that for FET S2D4 $\left(5.3 \mathrm{~cm}^{2} \mathrm{~s}^{-1} \mathrm{~V}^{-1}\right)$, reflecting scattering due to the GB between electrodes 2 and 4. FET S2D3 also showed an ON/OFF ratio of $\sim 10^{5}$. For the S1D5 FET the carrier mobility was calculated as $3.5 \mathrm{~cm}^{2} \mathrm{~s}^{-1} \mathrm{~V}^{-1}$. An FET device based on monolayer $\mathrm{WSe}_{2}$ without GBs was also fabricated using a bilayer flake with a large monolayer region (Figure S10a). This FET exhibited $p$-type behavior with a carrier mobility of $4.3 \mathrm{~cm}^{2} \mathrm{~s}^{-1} \mathrm{~V}^{-1}$ and an ON/OFF ratio of $\sim 5 \times 10^{4}$ (Figure $\mathrm{S} 10 \mathrm{~b}$ ). The results reflected the lower mobility of monolayer compared to the bilayer ${ }^{8}$ and further demonstrated the scattering caused by GBs. The relatively low ON/OFF ratios of the fabricated devices are ascribed to the relatively large OFF-state current $\left(10^{-10}-10^{-11} \mathrm{~A}\right)$, which reflects limitations of the instrument, since an OFF current of $\sim 10^{-12} \mathrm{~A}$ is frequently observed for similar back-gated $\mathrm{WSe}_{2} \mathrm{FETs}^{37,} 57$ The $\mathrm{I}_{\mathrm{ds}}-\mathrm{V}_{\mathrm{ds}}$ curves of the monolayer WSe 2 FET at varied gate voltages $\left(\mathrm{V}_{\mathrm{g}}\right)$ confirmed the $p$-type behavior of the material. A smaller current at negative $\mathrm{V}_{\mathrm{ds}}$ was recorded compared to 
the positive side, indicating the Schottky contact behavior. ${ }^{39}$ However, no significant rectification was observed, consistent with expectations (Figure S10c).

\section{CONCLUSION}

Controlled large-area CVD growth of high-density bilayer $\mathrm{WSe}_{2}$ flakes was achieved by using an optimized mixture of sodium cholate and sodium chloride as the growth promoter. The best bilayer morphology was obtained when the molar ratio of $\mathrm{C}_{24} \mathrm{H}_{40} \mathrm{NaO}_{5}$ to $\mathrm{NaCl}$ was 1:10. Further increase in the $\mathrm{NaCl}$ concentration was found to lead to the formation of multilayer $\mathrm{WSe}_{2}$ due to the improved diffusion of $\mathrm{W}$ precursors. The as-grown $\mathrm{WSe}_{2}$ mainly exhibited a six-point star-like morphology, with well-defined 1L-2L junctions. The majority of the bilayer regions $(\sim 84 \%)$ displayed twist angles of $0^{\circ}(\mathrm{AB})$ or $60^{\circ}\left(\mathrm{AA}^{\prime}\right)$, while moiré $15^{\circ}$ and $30^{\circ}$-twist angles were also observed. These CVD-grown $1 \mathrm{~L}-2 \mathrm{~L} \mathrm{WSe} \mathrm{W}_{2}$ junctions exhibited a $p-n$ diode characteristic with current rectification and an ambipolar transfer characteristic. Our results provide a method for the layer-controlled growth of TMDs, contributing to the fabrication of advanced atomically thin electronic and optoelectronic devices.

\section{METHODS}

\section{Growth of bilayer $\mathrm{WSe}_{2}$.}

Bilayer $\mathrm{WSe}_{2}$ flakes were grown directly on a $285 \mathrm{~nm} \mathrm{SiO} / 2 \mathrm{Si}$ substrate by CVD. First, a mixed aqueous solution of $25 \mathrm{mM}$ sodium cholate, $250 \mathrm{mM} \mathrm{NaCl}$, and $1.5 \mathrm{mM}$ ammonium metatungstate $\left(\mathrm{H}_{26} \mathrm{~N}_{6} \mathrm{O}_{40} \mathrm{~W}_{12}\right)$, is spin-coated at $4000 \mathrm{rpm}$ for $60 \mathrm{~s}$ onto the $\mathrm{SiO}_{2} / \mathrm{Si}$ substrate. After that, the substrate was placed in the center of a 1 inch CVD tube furnace and $150 \mathrm{mg}$ of selenium was placed $13 \mathrm{~cm}$ upstream from the substrate. Growth occurred at the atmospheric pressure in a flow of $350 \mathrm{sccm}$ of nitrogen gas (99.99\% purity) and $15 \mathrm{sccm}$ of $\mathrm{H}_{2}\left(99.999 \%\right.$ purity). The furnace temperature was ramped to $900{ }^{\circ} \mathrm{C}$ at a rate of $70{ }^{\circ} \mathrm{C}$ min ${ }^{-1}$. While the $\mathrm{SiO}_{2} / \mathrm{Si}$ growth substrate reached $900{ }^{\circ} \mathrm{C}$, the maximum temperature of the selenium was $\sim 220^{\circ} \mathrm{C}$. After a 10 min growth period, the furnace was opened, and the sample was rapidly cooled to room temperature in $700 \mathrm{sccm}$ flowing nitrogen. Growth with other concentrations of promoters, such as $25 \mathrm{mM} \mathrm{C}_{24} \mathrm{H}_{40} \mathrm{NaO}_{5}+125 \mathrm{mM} \mathrm{NaCl}, 25 \mathrm{mM}$ $\mathrm{C}_{24} \mathrm{H}_{40} \mathrm{NaO}_{5}+500 \mathrm{mM} \mathrm{NaCl}, 25 \mathrm{mM} \mathrm{NaCl}, 75 \mathrm{mM} \mathrm{NaCl}$, and $250 \mathrm{mM} \mathrm{NaCl}$, were conducted in a similar manner, respectively, to investigate the influence of growth promoters.

\section{Characterization.}

AFM analysis was conducted on an atomic force microscope (AFM, Icon Bruker) equipped with a probe with a tip radius of $<10 \mathrm{~nm}$ (TAP300AI-G, Budgetsensors) to evaluate the height profiles of bilayer $\mathrm{WSe}_{2}$. Raman and PL spectroscopy were performed under ambient conditions using an ND-MDT spectrometer equipped with a laser excitation wavelength of $532 \mathrm{~nm}$. For STEM imaging, bilayer $\mathrm{WSe}_{2}$ flakes were transferred onto a holy-carbon TEM grid using a poly(methyl methacrylate) (PMMA)-assisted transfer process. ${ }^{58}$ STEM was performed with a JEOL ARM 200CF equipped with a CEOS corrector (Cs $\sim 100 \mathrm{~nm}$ ) operating at $80 \mathrm{kV}$ to reduce knock-on damage. Images were acquired with a HAADF 
detector from 54-220 mrad and cleaned using an average background subtraction filter (ABSF) SAED patterns were acquired with a JEOL F200 TEM operating at $200 \mathrm{kV}$ using a selected-area aperture with an effective size at the sample of $\sim 1 \mu \mathrm{m}$.

\section{DFT calculations.}

First-principles DFT simulations are carried out using the Vienna ab initio simulation package (VASP) ${ }^{59}$. Projector-augmented wave pseudopotentials ${ }^{60}$ are used with a cutoff energy of $400 \mathrm{eV}$ for plane-wave expansions. The exchange-correlation is treated using the Perdew-Burke-Ernzerhof (PBE) generalized gradient approximations. ${ }^{61}$ The atomistic structures of monolayer and bilayer $\mathrm{WSe}_{2}$ are relaxed using $\Gamma$-centered $k$-point meshes of $18 \times 18 \times 1$ for primitive cells and $4 \times 4 \times 1$ for the twisted Moiré cells. The incommensurate Moiré cells with twist angles of $15^{\circ}$ and $30^{\circ}$ are strained $<0.4 \%$ into commensurate cells to satisfy periodic boundary conditions, and energetic corrections for the introduction of this strain are subsequently added. Long range van der Waals dispersion interactions were treated using the DFT-D3 method developed by Grimme et. al. ${ }^{62,63}$ For structural relaxations, the atomic positions of all unit and supercells are optimized until the force components on each atom are less than $0.005 \mathrm{eV} / \AA$, and the electronic energy is converged within $10^{-8} \mathrm{eV}$. A vacuum spacing of $20 \AA$ was added to prevent interactions between periodic images of the bilayers. Details of edge, substrate, and stacking energy determination can be found in the supporting information of our previous report. ${ }^{20}$

\section{Device fabrication.}

To fabricate FET devices for transport measurements, the as-grown bilayer $\mathrm{WSe}_{2}$ was spincoated with C4 PMMA (Microchem) under $3000 \mathrm{rpm}$ for $60 \mathrm{~s}$. Electron beam lithography (EBL) and plasma etching were used to define a rectangular region containing the $1 \mathrm{~L}$ and $2 \mathrm{~L}$ regions of the bilayer $\mathrm{WSe}_{2}$. Markers in the PMMA were defined by EBL, and then optical microscopy was used to determine the locations of rectangular flake with respect to the markers. A final round of EBL was performed to pattern contacts that were aligned to the $1 \mathrm{~L}$ and $2 \mathrm{~L}$ regions, and the contacts were metallized with $5 \mathrm{~nm} \mathrm{Cr}$ and $40 \mathrm{~nm}$ Au deposited by thermal evaporation. This was followed by lift-off in an acetone bath and a rinse in isopropyl alcohol (IPA). The sample was then dried by a $\mathrm{N}_{2}$ gun and transferred to the measurement system.

\section{Electrical measurements.}

Electrical measurements were performed under ambient condition in a probe station. Current-gate voltage $\left(\mathrm{I}_{\mathrm{ds}}-\mathrm{V}_{\mathrm{g}}\right)$ measurements were carried out using a Keithley 2400 sourcemeter, with a bias voltage of 1,2,3,4 V, respectively. The gate voltage was applied using a Keithley 6487 voltage source. The Current-bias voltage $\left(\mathrm{I}_{\mathrm{ds}}-\mathrm{Vd}_{\mathrm{s}}\right)$ measurements were carried out using a Keithley 6517A sourcemeter with different gate voltages.

\section{Supplementary Material}

Refer to Web version on PubMed Central for supplementary material. 


\section{ACKNOWLEDGMENT}

This work was supported by the NSF EFRI 2-DARE 1542879 and EAGER 1838412. P.M.D. and M.D. acknowledge support from NSF and NIH through the MRSEC DMR-1720530, NSF EFRI 2- DARE (EFRI-1542707), NSF EAGER 1838456, and NIH R21 HG010536, as well as the Penn Grant for Faculty Undergraduate Research Mentorship (FURM) supporting undergraduate research. V.B.S acknowledges support from Army Research Office contract W911NF-16-1- 0447 and NSF grants EFMA-542879 and CMMI-1727717. We thank Dr. Robert Keyse at Lehigh University for assistance with STEM imaging and Dr. Bing Hao for assistance with the figures. The authors acknowledge the use of the Raman system supported by NSF Major Research Instrumentation Grant DMR-0923245. This work was carried out in part at the Singh Center for Nanotechnology, part of the National Nanotechnology Coordinated Infrastructure Program, which is supported by the National Science Foundation grant NNCI-1542153.

\section{REFERENCES}

(1). Cai Z; Liu B; Zou X; Cheng H-M, Chemical Vapor Deposition Growth and Applications of TwoDimensional Materials and Their Heterostructures. Chem. Rev 2018, 118, 6091-6133. [PubMed: 29384374]

(2). Frisenda R; Molina-Mendoza AJ; Mueller T; Castellanos-Gomez A; van der Zant HSJ, Atomically Thin $P-N$ Junctions Based on Two-Dimensional Materials. Chem. Soc. Rev 2018, 47, 33393358. [PubMed: 29683464]

(3). Novoselov KS; Mishchenko A; Carvalho A; Castro Neto AH, 2D Materials and Van Der Waals Heterostructures. Science 2016, 353, aac9439. [PubMed: 27471306]

(4). Zeng Q; Liu Z, Novel Optoelectronic Devices: Transition-Metal-Dichalcogenide-Based 2D Heterostructures. Adv. Electron. Mater 2018, 4, 1700335.

(5). Wang H; Liu F; Fu W; Fang Z; Zhou W; Liu Z, Two-Dimensional Heterostructures: Fabrication, Characterization, and Application. Nanoscale 2014, 6, 12250-12272. [PubMed: 25219598]

(6). Wang X; Xia F, Stacked 2D Materials Shed Light. Nat. Mater 2015, 14, 264-265. [PubMed: 25643031]

(7). Geim AK; Grigorieva IV, Van Der Waals Heterostructures. Nature 2013, 499, 419-425. [PubMed: 23887427]

(8). Gong Y; Lin J; Wang X; Shi G; Lei S; Lin Z; Zou X; Ye G; Vajtai R; Yakobson BI; Terrones H; Terrones M; Tay Beng K.; Lou J; Pantelides ST; Liu Z; Zhou W; Ajayan PM, Vertical and InPlane Heterostructures from $\mathrm{WS}_{2} / \mathrm{MoS}_{2}$ Monolayers. Nat. Mater 2014, 13, 1135-1142. [PubMed: 25262094]

(9). Pomerantseva E; Gogotsi Y, Two-Dimensional Heterostructures for Energy Storage. Nat. Energy 2017, 2, 17089.

(10). Cheng R; Li D; Zhou H; Wang C; Yin A; Jiang S; Liu Y; Chen Y; Huang Y; Duan X, Electroluminescence and Photocurrent Generation from Atomically Sharp WSe $2 / \mathrm{MoS}_{2}$ Heterojunction $P-N$ Diodes. Nano Lett. 2014, 14, 5590-5597. [PubMed: 25157588]

(11). Fang H; Battaglia C; Carraro C; Nemsak S; Ozdol B; Kang JS; Bechtel HA; Desai SB; Kronast F; Unal AA; Conti G; Conlon C; Palsson GK; Martin MC; Minor AM; Fadley CS; Yablonovitch E; Maboudian R; Javey A, Strong Interlayer Coupling in Van Der Waals Heterostructures Built from Single-Layer Chalcogenides. Proc. Natl. Acad. Sci 2014, 111, 6198-6202. [PubMed: 24733906]

(12). Cao Y; Fatemi V; Demir A; Fang S; Tomarken SL; Luo JY; Sanchez-Yamagishi JD; Watanabe K; Taniguchi T; Kaxiras E; Ashoori RC; Jarillo-Herrero P, Correlated Insulator Behaviour at HalfFilling in Magic-Angle Graphene Superlattices. Nature 2018, 556, 80-84. [PubMed: 29512654]

(13). Cao Y; Fatemi V; Fang S; Watanabe K; Taniguchi T; Kaxiras E; Jarillo-Herrero P, Unconventional Superconductivity in Magic-Angle Graphene Superlattices. Nature 2018, 556, 43-50. [PubMed: 29512651]

(14). You J; Hossain MD; Luo Z, Synthesis of 2d Transition Metal Dichalcogenides by Chemical Vapor Deposition with Controlled Layer Number and Morphology. Nano Converg. 2018, 5, 26. [PubMed: 30467647] 
(15). Chhowalla M; Shin HS; Eda G; Li L-J; Loh KP; Zhang H, The Chemistry of Two-Dimensional Layered Transition Metal Dichalcogenide Nanosheets. Nature Chemistry 2013, 5, 263-275.

(16). Wang QH; Kalantar-Zadeh K; Kis A; Coleman JN; Strano MS, Electronics and Optoelectronics of Two-Dimensional Transition Metal Dichalcogenides. Nat. Nanotechnol 2012, 7, 699-712. [PubMed: 23132225]

(17). Xu M; Liang T; Shi M; Chen H, Graphene-Like Two-Dimensional Materials. Chem. Rev 2013, 113, 3766-3798. [PubMed: 23286380]

(18). He Y; Sobhani A; Lei S; Zhang Z; Gong Y; Jin Z; Zhou W; Yang Y; Zhang Y; Wang X; Yakobson B; Vajtai R; Halas NJ; Li B; Xie E; Ajayan P, Layer Engineering of 2D Semiconductor Junctions. Adv. Mater 2016, 28, 5126-5132. [PubMed: 27136275]

(19). Samad L; Bladow SM; Ding Q; Zhuo J; Jacobberger RM; Arnold MS; Jin S, Layer-Controlled Chemical Vapor Deposition Growth of $\mathrm{MoS}_{2}$ Vertical Heterostructures via Van Der Waals Epitaxy. ACS Nano 2016, 10, 7039-7046. [PubMed: 27373305]

(20). Ye H; Zhou J; Er D; Price CC; Yu Z; Liu Y; Lowengrub J; Lou J; Liu Z; Shenoy VB, Toward a Mechanistic Understanding of Vertical Growth of Van Der Waals Stacked 2D Materials: A Multiscale Model and Experiments. ACS Nano 2017, 11, 12780-12788. [PubMed: 29206441]

(21). Puretzky AA; Liang L; Li X; Xiao K; Sumpter BG; Meunier V; Geohegan DB, Twisted MoSe 2 Bilayers with Variable Local Stacking and Interlayer Coupling Revealed by Low-Frequency Raman Spectroscopy. ACS Nano 2016, 10, 2736-2744. [PubMed: 26762243]

(22). Liu G-B; Xiao D; Yao Y; Xu X; Yao W, Electronic Structures and Theoretical Modelling of TwoDimensional Group-VIB Transition Metal Dichalcogenides. Chem. Soc. Rev 2015, 44, 2643 2663. [PubMed: 25474725]

(23). Baugher BWH; Churchill HOH; Yang Y; Jarillo-Herrero P, Intrinsic Electronic Transport Properties of High-Quality Monolayer and Bilayer $\mathrm{MoS}_{2}$. Nano Lett. 2013, 13, 4212-4216. [PubMed: 23930826]

(24). Zhang Y; Tang T-T; Girit C; Hao Z; Martin MC; Zettl A; Crommie MF; Shen YR; Wang F, Direct Observation of a Widely Tunable Bandgap in Bilayer Graphene. Nature 2009, 459, 820-823. [PubMed: 19516337]

(25). Hong X; Kim J; Shi S-F; Zhang Y; Jin C; Sun Y; Tongay S; Wu J; Zhang Y; Wang F, Ultrafast Charge Transfer in Atomically Thin $\mathrm{MoS}_{2} / \mathrm{WS}_{2}$ Heterostructures. Nat. Nanotechnol 2014, 9 , 682-686. [PubMed: 25150718]

(26). Zhang C; Chen Y; Huang J-K; Wu X; Li L-J; Yao W; Tersoff J; Shih C-K, Visualizing Band Offsets and Edge States in Bilayer-Monolayer Transition Metal Dichalcogenides Lateral Heterojunction. Nat. Commun 2016, 7, 10349.

(27). Xu Z-Q; Zhang Y; Wang Z; Shen Y; Huang W; Xia X; Yu W; Xue Y; Sun L; Zheng C; Lu Y; Liao L; Bao Q, Atomically Thin Lateral $P-N$ Junction Photodetector with Large Effective Detection Area. 2D Mater. 2016, 3, 041001.

(28). Kim H; Yoon Y-G; Ko H; Kim SM; Rho H, Charge Transfer across Monolayer/Bilayer $\mathrm{MoS}_{2}$ Lateral Interface and Its Influence on Exciton and Trion Characteristics. 2D Mater. 2019, 6, 025004.

(29). Chu T; Ilatikhameneh H; Klimeck G; Rahman R; Chen Z, Electrically Tunable Bandgaps in Bilayer $\mathrm{MoS}_{2}$. Nano Lett. 2015, 15, 8000-8007. [PubMed: 26560813]

(30). Park S; Kim H; Kim MS; Han GH; Kim J, Dependence of Raman and Absorption Spectra of Stacked Bilayer $\mathrm{MoS}_{2}$ on the Stacking Orientation. Opt. Express 2016, 24, 21551-21559. [PubMed: 27661893]

(31). Withers F; Yang H; Britnell L; Rooney AP; Lewis E; Felten A; Woods CR; Sanchez Romaguera V; Georgiou T; Eckmann A; Kim YJ; Yeates SG; Haigh SJ; Geim AK; Novoselov KS; Casiraghi C, Heterostructures Produced from Nanosheet-Based Inks. Nano Lett. 2014, 14, 3987-3992. [PubMed: 24871927]

(32). Zhao M-Q; Trainor N; Ren CE; Torelli M; Anasori B; Gogotsi Y, Scalable Manufacturing of Large and Flexible Sheets of MXene/Graphene Heterostructures. Adv. Mater. Technol 2019, 4, 1800639. 
(33). Jeon J; Jang SK; Jeon SM; Yoo G; Jang YH; Park J-H; Lee S, Layer-Controlled CVD Growth of Large-Area Two-Dimensional $\mathrm{MoS}_{2}$ Films. Nanoscale 2015, 7, 1688-1695. [PubMed: 25385535]

(34). Lin Y-C; Jariwala B; Bersch BM; Xu K; Nie Y; Wang B; Eichfeld SM; Zhang X; Choudhury TH; Pan Y; Addou R; Smyth CM; Li J; Zhang K; Haque MA; Fölsch S; Feenstra RM; Wallace RM; Cho K; Fullerton-Shirey SK; Redwing JM; Robinson JA, Realizing Large-Scale, ElectronicGrade Two-Dimensional Semiconductors. ACS Nano 2018, 12, 965-975. [PubMed: 29360349]

(35). Yu Y; Li C; Liu Y; Su L; Zhang Y; Cao L, Controlled Scalable Synthesis of Uniform, HighQuality Monolayer and Few-Layer MoS 2 Films. Sci. Rep 2013, 3, 1866. [PubMed: 23689610]

(36). Zhang X; Choudhury TH; Chubarov M; Xiang Y; Jariwala B; Zhang F; Alem N; Wang G-C; Robinson JA; Redwing JM, Diffusion-Controlled Epitaxy of Large Area Coalesced WSe 2 Monolayers on Sapphire. Nano Lett. 2018, 18, 1049-1056. [PubMed: 29342357]

(37). Liu B; Fathi M; Chen L; Abbas A; Ma Y; Zhou C, Chemical Vapor Deposition Growth of Monolayer $\mathrm{WSe}_{2}$ with Tunable Device Characteristics and Growth Mechanism Study. ACS Nano 2015, 9, 6119-6127. [PubMed: 26000899]

(38). Xia M; Li B; Yin K; Capellini G; Niu G; Gong Y; Zhou W; Ajayan PM; Xie Y-H, Spectroscopic Signatures of $\mathrm{AB}^{\prime}$ and $\mathrm{AB}$ Stacking of Chemical Vapor Deposited Bilayer $\mathrm{MoS}_{2}$. ACS Nano 2015, 9, 12246-12254. [PubMed: 26536495]

(39). Kwak D-H; Jeong M-H; Ra H-S; Lee A-Y; Lee J-S, Lateral WSe $2 P-N$ Junction Device Electrically Controlled by a Single-Gate Electrode. Adv. Opt. Mater 2019, 7, 1900051.

(40). Ling X; Lee Y-H; Lin Y; Fang W; Yu L; Dresselhaus MS; Kong J, Role of the Seeding Promoter in $\mathrm{MoS}_{2}$ Growth by Chemical Vapor Deposition. Nano Lett. 2014, 14, 464-472. [PubMed: 24475747]

(41). Kim S-Y; Kwak J; Ciobanu CV; Kwon S-Y, Recent Developments in Controlled Vapor-Phase Growth of 2D Group 6 Transition Metal Dichalcogenides. Adv. Mater 2019, 31, 1804939.

(42). Liang L; Hu W; Xue Z; Shen J-W, Theoretical Study on the Interaction of Nucleotides on TwoDimensional Atomically Thin Graphene and Molybdenum Disulfide. FlatChem 2017, 2, 8-14.

(43). Li S; Wang S; Tang D-M; Zhao W; Xu H; Chu L; Bando Y; Golberg D; Eda G, Halide-Assisted Atmospheric Pressure Growth of Large $\mathrm{WSe}_{2}$ and $\mathrm{WS}_{2}$ Monolayer Crystals. Appl. Mater. Today 2015, 1, 60-66.

(44). Zhang K; Bersch BM; Zhang F; Briggs NC; Subramanian S; Xu K; Chubarov M; Wang K; Lerach JO; Redwing JM; Fullerton-Shirey SK; Terrones M; Robinson JA, Considerations for Utilizing Sodium Chloride in Epitaxial Molybdenum Disulfide. ACS Appl. Mater. Interfaces 2018, 10, 40831-40837. [PubMed: 30384598]

(45). Li H; Wu J; Huang X; Lu G; Yang J; Lu X; Xiong Q; Zhang H, Rapid and Reliable Thickness Identification of Two-Dimensional Nanosheets Using Optical Microscopy. ACS Nano 2013, 7, 10344-10353. [PubMed: 24131442]

(46). Wang J; Cai X; Shi R; Wu Z; Wang W; Long G; Tang Y; Cai N; Ouyang W; Geng P; Chandrashekar BN; Amini A; Wang N; Cheng C, Twin Defect Derived Growth of Atomically Thin $\mathrm{MoS}_{2}$ Dendrites. ACS Nano 2018, 12, 635-643. [PubMed: 29253328]

(47). Zheng W; Qiu Y; Feng W; Chen J; Yang H; Wu S; Jia D; Zhou Y; Hu P, Controlled Growth of Six-Point Stars $\operatorname{Mos}_{2}$ by Chemical Vapor Deposition and Its Shape Evolution Mechanism. Nanotechnology 2017, 28, 395601. [PubMed: 28671106]

(48). Rong Y; He K; Pacios M; Robertson AW; Bhaskaran H; Warner JH, Controlled Preferential Oxidation of Grain Boundaries in Monolayer Tungsten Disulfide for Direct Optical Imaging. ACS Nano 2015, 9, 3695-3703. [PubMed: 25870912]

(49). van der Zande AM; Huang PY; Chenet DA; Berkelbach TC; You Y; Lee G-H; Heinz TF; Reichman DR; Muller DA; Hone JC, Grains and Grain Boundaries in Highly Crystalline Monolayer Molybdenum Disulphide. Nat. Mater 2013, 12, 554-561. [PubMed: 23644523]

(50). Zeng H; Liu G-B; Dai J; Yan Y; Zhu B; He R; Xie L; Xu S; Chen X; Yao W; Cui X, Optical Signature of Symmetry Variations and Spin-Valley Coupling in Atomically Thin Tungsten Dichalcogenides. Sci. Rep 2013, 3, 1608. [PubMed: 23575911] 
(51). Zhao W; Ghorannevis Z; Chu L; Toh M; Kloc C; Tan P-H; Eda G, Evolution of Electronic Structure in Atomically Thin Sheets of $\mathrm{WS}_{2}$ and $\mathrm{WSe}_{2}$. ACS Nano 2013, 7, 791-797. [PubMed: 23256505]

(52). Thiruraman JP; Fujisawa K; Danda G; Das PM; Zhang T; Bolotsky A; Perea-López N; Nicolaï A; Senet P; Terrones M; Drndić M, Angstrom-Size Defect Creation and Ionic Transport through Pores in Single-Layer $\mathrm{MoS}_{2}$. Nano Lett. 2018, 18, 1651-1659. [PubMed: 29464959]

(53). Alexeev EM; Ruiz-Tijerina DA; Danovich M; Hamer MJ; Terry DJ; Nayak PK; Ahn S; Pak S; Lee J; Sohn JI; Molas MR; Koperski M; Watanabe K; Taniguchi T; Novoselov KS; Gorbachev RV; Shin HS; Fal'ko VI; Tartakovskii AI, Resonantly Hybridized Excitons in Moiré Superlattices in Van Der Waals Heterostructures. Nature 2019, 567, 81-86. [PubMed: 30842637]

(54). Rakhmanov AL; Rozhkov AV; Sboychakov AO; Nori F, Instabilities of the Aa-Stacked Graphene Bilayer. Phys. Rev. Lett 2012, 109, 206801. [PubMed: 23215515]

(55). Xia J; Yan J; Shen ZX, Transition Metal Dichalcogenides: Structural, Optical and Electronic Property Tuning via Thickness and Stacking. FlatChem 2017, 4, 1-19.

(56). Yan A; Chen W; Ophus C; Ciston J; Lin Y; Persson K; Zettl A, Identifying Different Stacking Sequences in Few-Layer Cvd-Grown $\mathrm{MoS}_{2}$ by Low-Energy Atomic-Resolution Scanning Transmission Electron Microscopy. Phys. Rev. B 2016, 93, 041420.

(57). Gao Y; Hong Y-L; Yin L-C; Wu Z; Yang Z; Chen M-L; Liu Z; Ma T; Sun D-M; Ni Z; Ma X-L; Cheng H-M; Ren W, Ultrafast Growth of High-Quality Monolayer WSe 2 on Au. Adv. Mater 2017, 29, 1700990.

(58). Naylor CH; Parkin WM; Ping J; Gao Z; Zhou YR; Kim Y; Streller F; Carpick RW; Rappe AM; Drndić M; Kikkawa JM; Johnson ATC, Monolayer Single-Crystal 1T'-MoTe 2 Grown by Chemical Vapor Deposition Exhibits Weak Antilocalization Effect. Nano Lett. 2016, 16, 4297 4304. [PubMed: 27223343]

(59). Kresse G; Furthmüller J, Efficient Iterative Schemes for Ab Initio Total-Energy Calculations Using a Plane-Wave Basis Set. Phys. Rev. B 1996, 54, 11169-11186.

(60). Blöchl PE, Projector Augmented-Wave Method. Phys. Rev. B 1994, 50, 17953-17979.

(61). Perdew JP; Burke K; Ernzerhof M, Generalized Gradient Approximation Made Simple. Phys. Rev. Lett 1996, 77, 3865-3868. [PubMed: 10062328]

(62). Grimme S; Antony J; Ehrlich S; Krieg H, A Consistent and Accurate Ab Initio Parametrization of Density Functional Dispersion Correction (Dft-D) for the 94 Elements H-Pu. J. Chem. Phys 2010, 132, 154104. [PubMed: 20423165]

(63). Grimme S; Ehrlich S; Goerigk L, Effect of the Damping Function in Dispersion Corrected Density Functional Theory. J. Comput. Chem 2011, 32, 1456-1465. [PubMed: 21370243] 
(a)

(b)

(c)

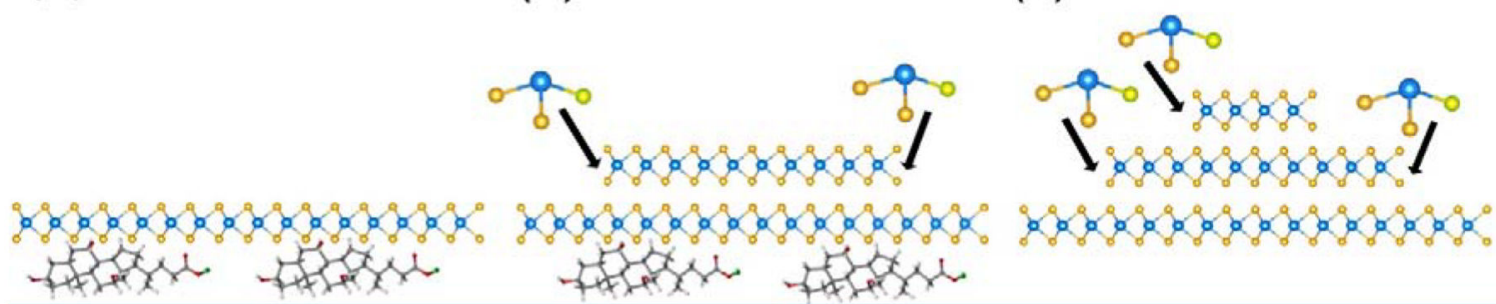

\section{Substrate}

- He

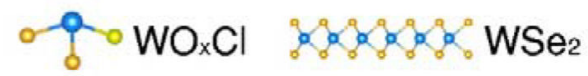

Figure 1.

Schematic showing how the choice of promoter affects CVD growth of $\mathrm{WSe}_{2}$ : (a) Sodium cholate promoter leads to growth of monolayer $\mathrm{WSe}_{2}$; (b) The correct mixture of sodium cholate and $\mathrm{NaCl}$ leads to the formation of volatile oxyhalides $\left(\mathrm{WO}_{\mathrm{x}} \mathrm{Cl}\right)$ and enhanced growth of bilayer $\mathrm{WSe}_{2}$; (c) Pure $\mathrm{NaCl}$ promoter leads to an overabundance of oxyhalides and growth of multilayer $\mathrm{WSe}_{2}$. 

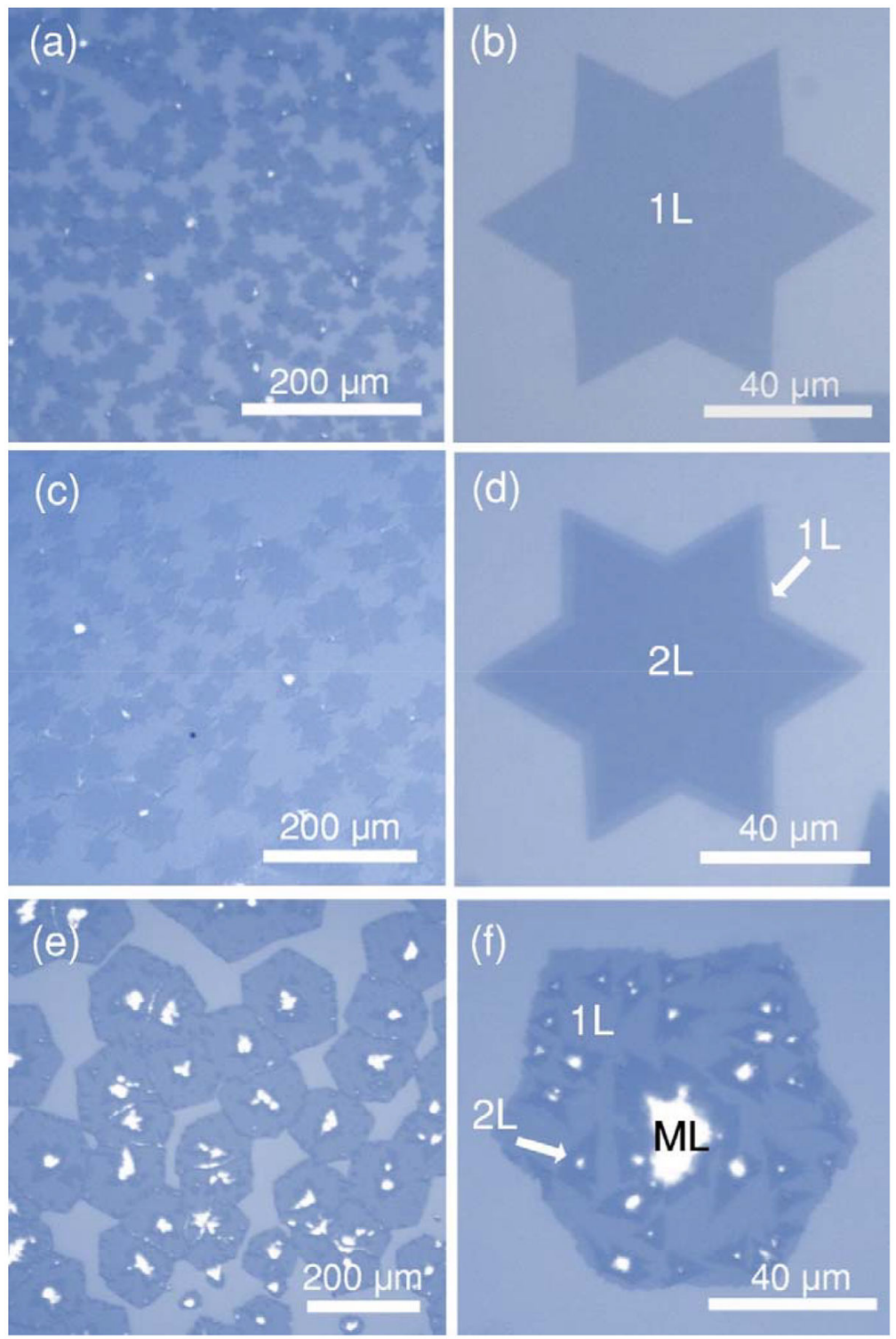

Figure 2.

Optical micrographs showing the morphology of as-grown $\mathrm{WSe}_{2}$ flakes. (a,b) Use of pure sodium cholate as promoter leads to the formation of monolayer flakes. (c,d) A 1:10 mixture of sodium cholate and $\mathrm{NaCl}$ as promoter leads to a high coverage of bilayer flakes. (e,f) Use of pure $\mathrm{NaCl}$ as the promoter leads to predominantly multi-layer flakes. 

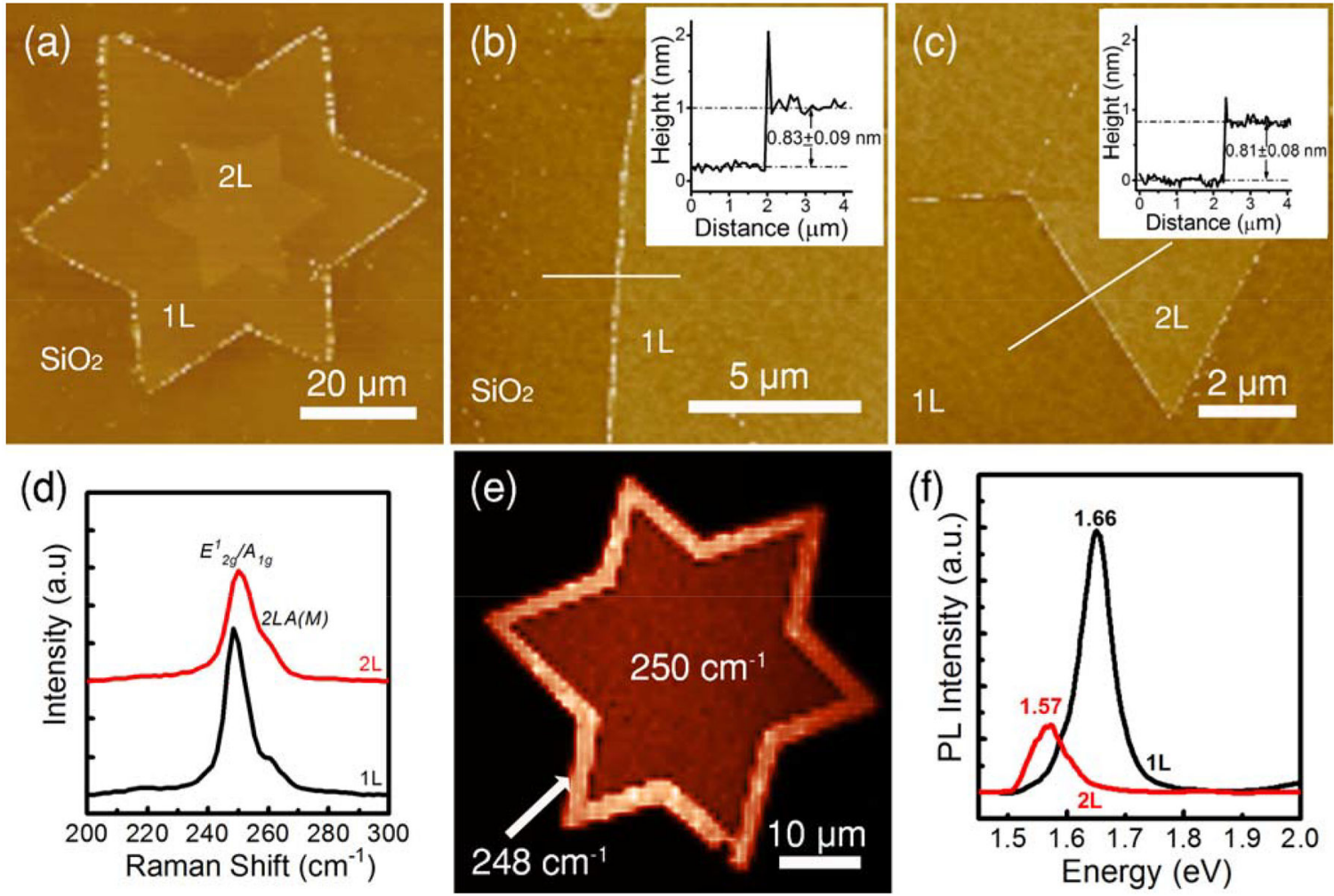

Figure 3.

Atomic Force Microscope images showing (a) a bilayer $\mathrm{WSe}_{2}$ flake on a $\mathrm{SiO}_{2}$ substrate and a zoom in on the (b) substrate-to-1L and (c) 1L-to-2L regions. The measured height values are shown in the corresponding insets. (d) Raman spectra of the $1 \mathrm{~L}$ and $2 \mathrm{~L}$ regions of bilayer $\mathrm{WSe}_{2}$; (e) Combined Raman intensity map at 248 and $250 \mathrm{~cm}^{-1}$ for a bilayer $\mathrm{WSe}_{2}$ flake; (d) Photoluminescence spectra collected from the $1 \mathrm{~L}$ and $2 \mathrm{~L}$ regions of a bilayer $\mathrm{WSe}_{2}$ flake. 

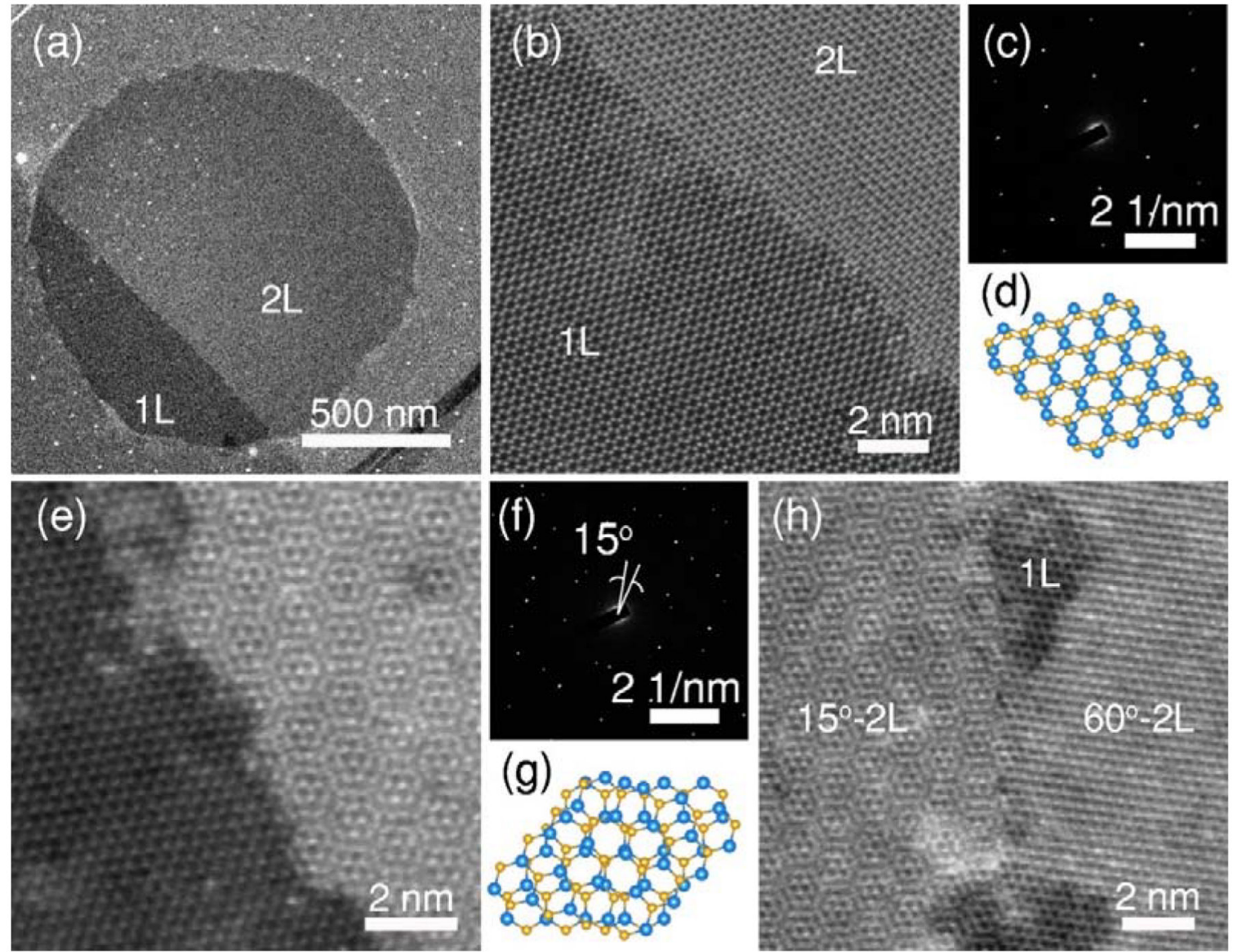

Figure 4.

(a) Low-magnification scanning transmission electron microscope (STEM) image showing a 1L-2L junction in a CVD-grown bilayer $\mathrm{WSe}_{2}$ flake; (b) STEM high-angle annular dark field (STEM-HAADF) image showing a 1L-2L boundary with a $60^{\circ}$ (AA')-twist angle in the $2 \mathrm{~L}$ region with (c) corresponding SAED pattern and (d) an atomic model; (e) STEMHAADF image showing a $1 \mathrm{~L}-2 \mathrm{~L}$ boundary with a $15^{\circ}$-twist angle in the $2 \mathrm{~L}$ region with (f) corresponding SAED pattern and (g) an atomic model; (h) STEM-HAADF image showing the $2 \mathrm{~L}$ region with a $\mathrm{GB}$ of a star-like bilayer $\mathrm{WSe}_{2}$ flake, in which 15 and $60^{\circ}$-twist angles are observed at the two sides of the GB, respectively. 

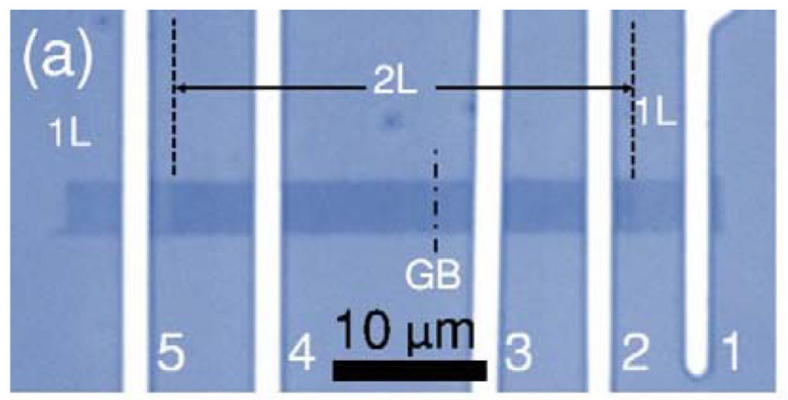

(b)

$4 \quad G B \quad 3 \quad 2$

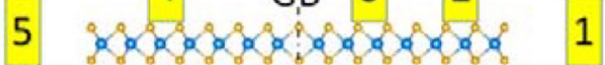

$80000000000000000 \%$

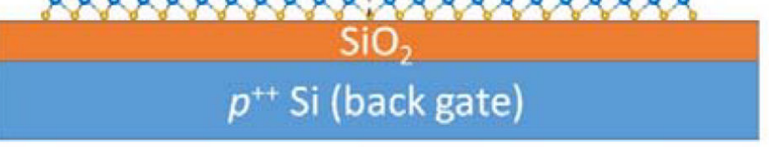

(c)

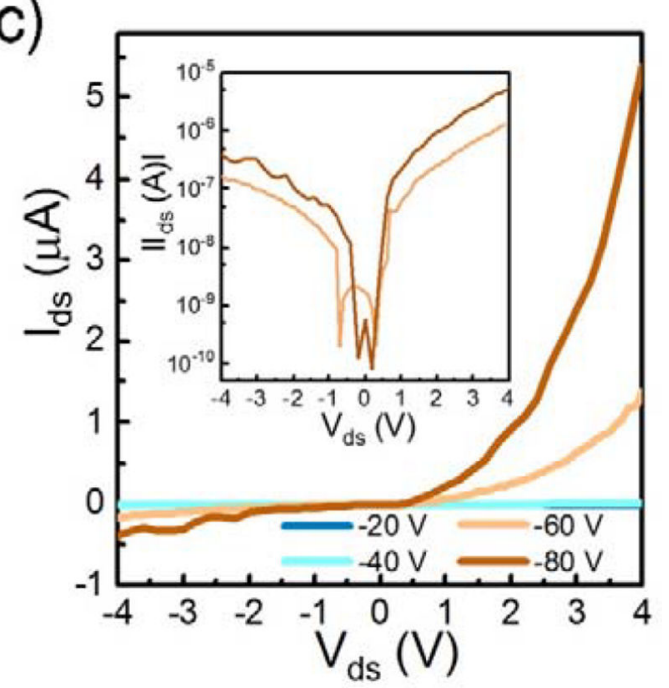

(d)
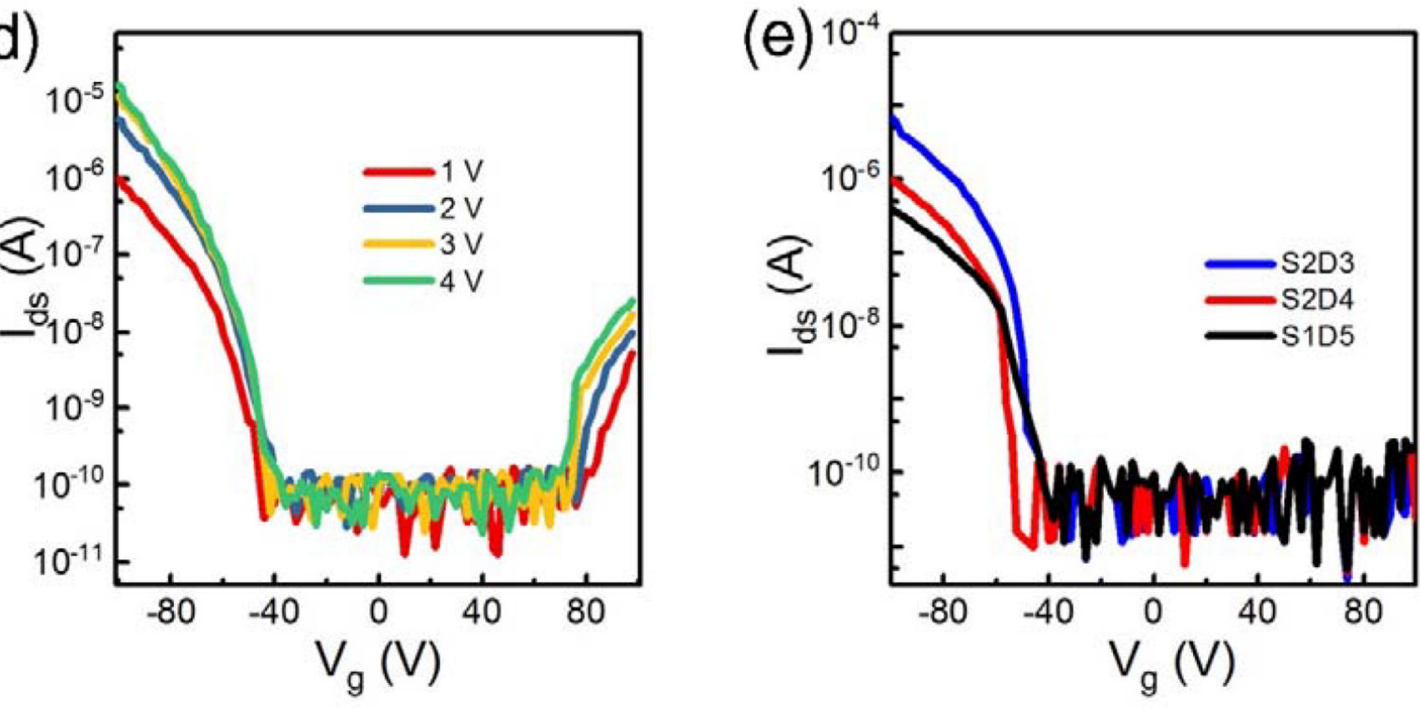

Figure 5.

(a) Optical micrograph and (b) schematic of the fabricated set of FET devices based on asgrown bilayer $\mathrm{WSe}_{2}$; (c) $\mathrm{I}_{\mathrm{ds}}-\mathrm{V}_{\mathrm{ds}}$ curves at different $\mathrm{V}_{\mathrm{g}}$ of the $\mathrm{WSe}_{2}$-based FET with a 1L-2L junction (S1D2); The inset shows the same data on a logarithmic scale. (d) $\mathrm{I}_{\mathrm{ds}}-\mathrm{V}_{\mathrm{g}}$ curves at different $\mathrm{V}_{\mathrm{ds}}$ of the $\mathrm{WSe}_{2}$-based FET with a 1L-2L junction (S1D2); (e) Comparison of the $\mathrm{I}_{\mathrm{ds}}-\mathrm{V}_{\mathrm{g}}$ curves for bilayer WSe $\mathrm{W}_{2}$-without GBs (S2D3), bilayer WSe 2 with a GB (S2D4), and monolayer $\mathrm{WSe}_{2}$ with a GB (S1D5) based FET devices. 


\section{Table 1}

Calculated vdW stacking energy density for different orientations of $\mathrm{WSe}_{2}$ bilayers, along with lattice constants used.

\begin{tabular}{c|c|c|c|c|c}
\hline & $\mathbf{0}^{\circ}(\mathbf{A A})$ & $\mathbf{0}^{\circ}(\mathbf{A B})$ & $\mathbf{1 5}^{\circ}$ (Moiré) & $\mathbf{3 0}^{\circ}$ (Moiré) & $\mathbf{6 0}^{\circ}\left(\mathbf{A A}^{\prime}\right)$ \\
\hline $\begin{array}{c}\varepsilon_{\mathrm{L} 1 \mathrm{~L} 2} \\
\left(\mathrm{meV} / \AA^{2}\right)\end{array}$ & -23.3 & -34.3 & -28.9 & -29.3 & -35.0 \\
$a(\AA)$ & 3.29 & 3.29 & 21.54 & 22.87 & 3.29 \\
\hline
\end{tabular}

